\title{
A Chosen Plaintext Steganalysis of Hide4PGP V 2.0
}

\author{
Debasis Mazumdar ${ }^{1}$, Soma Mitra ${ }^{1}$, Sonali Dhali ${ }^{1}$, and Sankar K. Pal ${ }^{2}$ \\ ${ }^{1}$ CDAC,Kolkata, Plot E2/1, Block - GP, Sector V, Salt Lake City, Kolkata - 700091 \\ ${ }^{2}$ Machine Intelligence Unit, Indian Statistical Institute, Kolkata - 700108, India
}

\begin{abstract}
A chosen plaintext steganalysis algorithm is described to isolate the corrupted bits in an image tampered with Hide4PGP V 2.0. The method is developed from the notion of representation of two dimensional image data in terms of a linear bit stream consisting of a set of basic building blocks. Its performance for message extraction is demonstrated on different 24 bit BMP images.
\end{abstract}

\section{Introduction}

Hide4PGP V 2.0 is a steganography tool, which is readily available and used frequently to tamper both image and audio files. It encrypts the message before distribution; thereby making the tool robust. Some schemes are reported in this regard based on statistical measures [1] and pallet color [2]. First and higher order statistics are also used to discriminate between images with and without hidden messages using LSB embedding stego tools like Hide4PGP ([3],[4]). M.K.Johnson et. al. [5] has used frequency domain analysis for audio signals to detect the existence of hidden message embedded using Hide4PGP. However, these methods can only detect the presence or absence of hidden message within a cover. In the present article, we describe a steganalysis algorithm based on chosen plaintext attacking scheme, which can identify and isolate the corrupted bits. The isolation of corrupted bits helps us to get back the ciphertext. To reconstruct the plaintext message out of the ciphertext one may use further cryptanalysis. The algorithm uses a unique method of representing two dimensional image data as a linear bit string consisting of a set of basic building blocks. The effectiveness of the method is demonstrated on a set of 24 bit BMP images of varying sizes.

\section{Characteristics of Message Distribution}

After a series of experiments using monotonic (single color) cover image (having $\mathrm{p}$ no. of pixels) and known plaintext we found the following characteristics (patterns) of the distribution used in embedding a message in $3 p$ number of LSB positions.

i) The message, of length $m_{L}$, is embedded symmetrically within the LSBs of R, G and $\mathrm{B}$ components of $\mathrm{p}$ number of pixels. These LSBs, when arranged in a linear string, shows reflection symmetry with respect to centre position. Also in each part the arrangement is same when read from left to right or vice-versa. These are 
illustrated in (Fig. 2), where the black and white squares represent tampered and untampered bits respectively.

ii) Each part of the string, if further divided into two equal parts, the same symmetrical properties, as stated above, are observed. This continues up to a critical segment of the string beyond which its further splitting does not result in the said symmetrical property. Let this segment be defined as minimum unit $\mathrm{M}$ of length $\boldsymbol{\zeta}_{\mathrm{M}}$.

iii) Untampered ones separate tampered LSBs. No two tampered bits can occur together. Number of white squares (i.e. untampered bits) separating two black squares is variable. Let this number of such consecutive white squares be called the run length $(\mathcal{l})$.

iv) In any tampered image, there exist only two types of run lengths (even and odd), and they differ in length only by unity. The run length depends on both $\mathrm{p}$ and $\mathrm{m}_{\mathrm{L}}$.

Note that, all the aforesaid characteristics are valid only when $m_{L}<3 p / 2$. If $m_{L}>$ $3 \mathrm{p} / 2$, the same properties hold good with the black and white squares interchanged. When $\mathrm{m}_{\mathrm{L}}=3 \mathrm{p} / 2$, the LSB positions are alternatively occupied by the message bits and there exists only one type of run length of value unity. Beside these, the following special cases may be noted regarding message embedding.

Case I: $\mathrm{m}_{\mathrm{L}}=3 \mathrm{p}$, Here all the LSBs are tampered; i.e., only one type of run length, having zero value, exists.

Case II: $3 \mathrm{p}<\mathrm{m}_{\mathrm{L}} \leq 6 \mathrm{p}$, All the $3 \mathrm{p}$ LSBs get tampered first and then the remaining $\left(\mathrm{m}_{\mathrm{L}}-3 \mathrm{p}\right)$ message bits are distributed in the next significant $3 \mathrm{p}$ bits following the aforesaid symmetrical characteristics ((i) - (iv) ).

Case III: $\mathrm{m}_{\mathrm{L}}>6 \mathrm{p}$, Hide4PGP V 2.0 fails to embed the message.

\subsection{Linear Bit-String and Basic Building Blocks}

A linear bit string consisting of tampered (black squares) and untampered bits (white squares), as shown in Fig. 2, can be viewed as an ensemble of basic building blocks, where each block consists of three elements nomenclatured as black ball $(\bullet)$, white ball (o), and the message bit ( $\bullet$ ) of unit length. Black ball represents even run length l. White ball represents odd run length $\mathcal{l}+1$ (or $\mathcal{l}-1$ ). Depending on the combinations of black ball, white ball and message bit, different building blocks such as, $\mathrm{u}_{1}, \mathrm{u}_{2}, \mathrm{u}_{2 \mathrm{R}}$, $\mathrm{u}_{3}, \mathrm{u}_{4}$ and $\mathrm{u}_{5}$ can be defined, as shown in Fig 1 .

\begin{tabular}{|c|c|c|}
\hline Basic building block & Structure & Lengths in Bits \\
\hline $\mathrm{u}_{1}$ & $\diamond \mathrm{०}$ & $\mathcal{l}+3($ or $l+1)$ \\
\hline $\mathrm{u}_{2}$ & $\bullet \bullet$ & $2 l+2($ or $2 l)$ \\
\hline $\mathrm{u}_{2 \mathrm{R}}$ & $0 \bullet \bullet$ & $2 \mathcal{l}+2($ or $2 \ell)$ \\
\hline $\mathrm{u}_{3}$ & $\diamond \bullet \bullet$ & $\lceil+2$ \\
\hline $\mathrm{u}_{4}$ & $\mathrm{O} \diamond \mathrm{O}$ & $2 \complement+3($ or $2 \ell-1)$ \\
\hline $\mathrm{u}_{5}$ & $\bullet \bullet \bullet$ & $2 \complement+1$ \\
\hline
\end{tabular}

Fig. 1. Different basic building blocks and their lengths in bits 


\section{Method of Message Extraction}

Let $\xi$ be a tampered bit string containing $\mathrm{n}$ number of minimum units represented by the building blocks as shown in Fig. 2 .

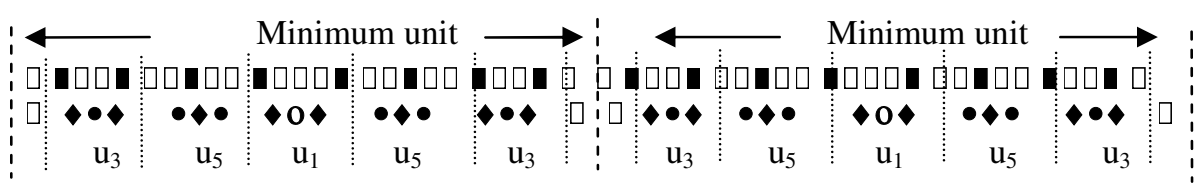

Fig. 2. Representation of minimum unit length of a bit string in terms of basic building blocks

Here all the diamonds $(\bullet)$ in the $\mathrm{n}$ minimum units contain the hidden message. Therefore, determining their locations is necessary for extracting the message. In order to do that, the following steps may be taken:

Step 1: Compute the minimum unit length $\varsigma_{\mathrm{M}}$ in $\xi$.

Step 2: Compute all possible combinations of $\mathrm{u}_{1}, \mathrm{u}_{2}, \mathrm{u}_{2 \mathrm{R}}, \mathrm{u}_{3}$ and $\mathrm{u}_{4}\left(\right.$ or $\left.\mathrm{u}_{5}\right)$ as determined by their number of occurrences within $\mathrm{l}_{\mathrm{M}}$.

Step 3: Determine that combination(s) of the building blocks within $\mathfrak{l}_{\mathrm{M}}$, which shows the symmetry property.

Step 4: Isolate the diamond bits $(\diamond)$ in all the $\mathrm{n}$ minimum units, arrange them linearly and extract the message contained in the string $\xi$ through decryption using the descrambler [6].

In certain cases $\xi$ may have more than one combination of building blocks having symmetry property. In that case, except one other would be (syntactically/ semantically) incorrect.

In the following sections we explain the methods of computing the length $\left(\mathcal{G}_{M}\right)$ of the minimum unit (Step 1), determining the number of basic building blocks in the minimum unit (Step 2), detecting the combinations of the building blocks which posses the symmetry (Step 3), and extracting the message bits from the entire string $\xi$ (Step 4).

\subsection{Computation of Length of Minimum Unit}

Within the linear bit string $\xi$ having $3 p$ number of available LSBs, let, $\mathrm{m}_{\mathrm{L}}$ message bits be distributed symmetrically. Let, in $\xi$, two consecutive message bits be separated by $q$ number of untampered bits. The difference between the total number of available LSBs (3p) in $\xi$ and the number of message bits $\mathrm{m}_{\mathrm{L}}$, therefore, gives the number of positions occupied by all the untampered bits. So, one can write:

$$
3 \mathrm{p}-\mathrm{m}_{\mathrm{L}}=\mathrm{qm}_{\mathrm{L}}
$$

For integral values of $\mathrm{p}$ and $\mathrm{m}_{\mathrm{L}}, \mathrm{q}$ may be either an integer or a real number. Since the number of untampered bits separating two message bits is always an integer, the adjustment (or justification) of the fractional part of $\mathrm{q}$ is made considering the occurrences of the even and odd run lengths of unit difference as follows: 
If floor of q (i.e., $\left\lfloor^{q}\right\rfloor$ ) is odd, then the even run length $l=\left\lfloor{ }^{q}\right\rfloor+1$ and the odd run length $l-1=\left\lfloor\left\lfloor^{\mathrm{q}}\right\rfloor\right.$. When $\left\lfloor^{\mathrm{q}}\right\rfloor$ is even, the even run length $l=\left\lfloor^{\mathrm{q}}\right\rfloor$ and the odd run length $l+1=\lfloor q\rfloor+1$. If $q$ is an integer, then obviously there exists only one type of run length (either even or odd) separating two consecutive message bits. Let there be $\mathrm{x}$ number of black balls and y number of white balls in $\xi$. Since a message bit always follows each of black and white balls, total number of message bits is equal to the sum of the number of black and white balls. Thus,

$$
\mathrm{x}+\mathrm{y}=\mathrm{m}_{\mathrm{L}}
$$

In terms of $\mathrm{x}$ and $\mathrm{y}$, and the even and odd run lengths, Eq. (1) can be written as,

$$
3 \mathrm{p}-\mathrm{m}_{\mathrm{L}}=\ell \mathrm{x}+(\mathcal{l} \pm 1) \mathrm{y}
$$

' + ' stands for even run length $\mathcal{l}$ and odd run length $\mathcal{l}+1$ while '-' stands for even run length $\ell$ and odd run length $\mathcal{l}-1$.

Solving the above linear equations for $\mathrm{x}$ and $\mathrm{y}$, one can compute the ratio $(\mathrm{k})$ of the number of black balls to the number of white balls in the entire string $\xi$.

Since the data distribution in $\xi$ follows the symmetrical properties (described in Section 2), the value of k computed over the entire string $\xi$ would be the same even if computed within the minimum unit $\widehat{l}_{\mathrm{M}}$. Let, $\mathrm{b}_{\mathrm{b}}$ and $\mathrm{b}_{\mathrm{w}}$ be the number of black and white balls in the minimum unit length $\widehat{c}_{\mathrm{M}}$. Then,

$$
\mathrm{b}_{\mathrm{b}} / \mathrm{b}_{\mathrm{w}}=\mathrm{k}
$$

where $b_{b}$ and $b_{w}$ represent two smallest possible integers satisfying Eq. (4).

Therefore, the length of the minimum unit $f_{M}$ can be computed as:

$$
c_{\mathrm{M}}=\mathrm{b}_{\mathrm{w}}(\mathcal{l}(1+\mathrm{k})+\mathrm{k})
$$

After obtaining $\mathfrak{l}_{\mathrm{M}}$, the next task is to determine all possible combinations of the basic building blocks $\mathrm{u}_{\mathrm{i}} \mathrm{s}$ in it (Step 2), and then find the one, which shows symmetry property (Step 3). In Section 3.2 we explain the way of computing all such combinations of $u_{i} s$ with different $n_{i}$ values in $l_{M}$. The method of determining the symmetrical sequence out of them is then described in Section 3.3.

\subsection{Computation of the Number of Basic Building Blocks in the Minimum Unit}

Let $n_{i}$ be the number of $i^{\text {th }}$ basic building block $u_{i}$ of length $\ell_{i}(i=1,2,2 R, 3,4$ and 5$)$ occurred in the minimum unit of length $f_{\mathrm{M}}$. While representing the minimum unit in terms of basic building blocks, as discussed in Section 2.1 and represented in Fig. 2, l 12 number of white squares are seen to remain unaccounted both at the beginning and end points of the minimum unit. Hence the remaining $\left(\mathcal{G}_{\mathrm{M}}-\ell\right)$ number of bits are represented by $n_{i}$ number of $u_{i}$ units. Therefore one can write,

$$
\sum \mathrm{n}_{\mathrm{i}} \ell_{\mathrm{i}}=l_{\mathrm{M}}-l
$$


Three independent equations can be derived from Eq.(6) expressing the number of black balls, white balls and message bits in $u_{i}$ in terms of the number of black balls $\left(b_{b}\right)$, white ballsc $\left(b_{w}\right)$ and message bits. In any minimum unit $\mathcal{l}_{M}$, five different $n_{i} s$, namely, $\left(\mathrm{n}_{1}, \mathrm{n}_{2}, \mathrm{n}_{2 \mathrm{R}}, \mathrm{n}_{3}, \mathrm{n}_{4}\right)$ or $\left(\mathrm{n}_{1}, \mathrm{n}_{2}, \mathrm{n}_{2 \mathrm{R}}, \mathrm{n}_{3}, \mathrm{n}_{5}\right)$ can occur. Since it involves computation of five variables from three equations, the algorithm will give degenerate solutions. Further, units $u_{2}$ and $u_{2 R}$ are of equal length; hence, although the algorithm needs to compute only one $\mathrm{n}_{\mathrm{i}}$ value for them, it is not possible to identify whether that value corresponds to $\mathrm{u}_{2}$ or $\mathrm{u}_{2 \mathrm{R}}$. Under the above circumstances, for a string $\xi$ having $\sum \mathrm{n}_{\mathrm{i}}$ number of basic building blocks, the total number of degenerate solutions is

$$
\mathrm{N}=\sum_{\mathrm{k}=1}^{\sum \mathrm{n}_{\mathrm{i}} / 2}\left(\left(\sum \mathrm{n}_{\mathrm{i}}+3-2 \mathrm{k}\right)^{2}\right) .
$$

Out of them, usually the distribution (arrangements) of $\mathrm{u}_{\mathrm{i}} \mathrm{s}$ corresponding to only one solution will posses the symmetry property, as stated in Section 2.

\subsection{Determination of Minimum Unit with Building Blocks}

In order to determine the solution(s) with symmetry property, one needs to check several conditions with the arrangements of $\mathrm{u}_{\mathrm{i}} \mathrm{s}$ in $l_{\mathrm{M}}$. To articulate them, let us divide the set of basic building blocks i.e., $\left\{\mathrm{u}_{1}, \mathrm{u}_{2}, \mathrm{u}_{2 \mathrm{R}}, \mathrm{u}_{3}, \mathrm{u}_{4}\left(\right.\right.$ or $\left.\left.\mathrm{u}_{5}\right)\right\}$ into two subsets, namely, $\mathrm{S}_{1}=\left\{\mathrm{u}_{1}, \mathrm{u}_{3}\right\}$ and $\mathrm{S}_{2}=\left\{\mathrm{u}_{2}, \mathrm{u}_{2 \mathrm{R}}, \mathrm{u}_{4}\left(\right.\right.$ or $\left.\left.\mathrm{u}_{5}\right)\right\}$. With $\mathrm{S}_{1}, \mathrm{~S}_{2}$ and the ratio $\mathrm{k}$ (Section 3.1), the conditions are stated below:

Condition 1: The even positions in the minimum unit $l_{\mathrm{M}}$ are always occupied by the elements of $S_{2}$ while elements of $S_{1}$ always occupy the odd positions.

Condition 2: The number of consecutive white balls and black balls can be determined depending on the value of $\mathrm{k}$, as follows:

i) If $\mathrm{k}>1$ and $\mathrm{k} \in \mathrm{Z}$ ( $\mathrm{Z}$ is the set of positive integers) then each white ball will be followed by maximum $k$ number of black balls. ii) If $k>1$ and $k \in R$ ( $R$ is the set of rational numbers) then each white ball will be followed by either $\lceil k\rceil$ or $\lfloor k\rfloor$ number of black balls. iii) If $\mathrm{k}<1$ and $1 / \mathrm{k} \in \mathrm{Z}$ then each black ball will be followed by maximum $\mathrm{k}$ number of white balls. iv) If $\mathrm{k}<1$ and $1 / \mathrm{k} \in \mathrm{R}$ then each black ball will be followed by either $\lceil 1 / \mathrm{k}\rceil$ or $\lfloor 1 / \mathrm{k}\rfloor$ numbers of white balls.

\subsection{Extraction of Message from $\xi$}

After obtaining the solutions having symmetry property from the previous phase (Section 3.3), perform the following tasks:

i) Select one such solution. ii) Repeat it so as to construct the entire string $\xi$. iii) Isolate all the diamond $(\bullet)$ bits and put them consecutively to form a cipher string. iv) Decrypt the cipher string using the Descrambler function [6]. v) Check the decrypted message (text) syntactically/semantically, and accept it as the correct message. Otherwise select another solution with symmetry property and perform the above tasks (ii - iv). 


\section{Experimental Results}

To demonstrate the effectiveness of our algorithm, two hundred 24-bit BMP images of different sizes, ranging from $(8 \times 8)$ to $(512 \times 512)$ pixels, are tampered using Hide4PGP V 2.0. The hidden text message is of different lengths covering 2 to $25 \%$ of the cover image. All these tampered images are then decrypted with the method described in Section 3. In all the cases the algorithm has been able to extract the hidden message.

\section{Conclusions}

We have described an attacking algorithm for extraction of hidden message out of stego images created using Hide4PGP V 2.0. The effectiveness of the methodology is demonstrated over a large number of 24 bit BMP images of different sizes. This algorithm is also applicable to WAV and VOC file formats tampered with Hide4PGP V 2.0.

\section{Acknowledgements}

The authors gratefully acknowledge the financial support through project no. 12(20)/04-IRSD of the DIT, MCIT, Govt. of India, New Delhi.

\section{References}

1. Dumitresu, S., Wu, X., Wang, Z.: Detection of LSB Steganography Via Sample Pair Analysis. Lecture Notes in Computer Science, Vol. 2578. Springer-Verlag, Noordwijkerhout, The Netherlands (2002) 355-372

2. Johnson, N.F., Jajodia, S.: Steganalysis of Images Created Using Current Steganography Software Lecture Notes in Computer Science, Vol. 1525. Springer-Verlag, Portland Oregon USA (1998) 273-289.

3. Lyu, S., Farid, H.: Detecting Hidden Messages Using Higher-Order Statistics and Support Vector Machines. Lecture Notes in Computer Science, Vol. 2578. Springer-Verlag, Noordwijkerhout, The Netherlands (2002) 340-354

4. Westfeld, A.: Detecting Low Embedding Rates. Lecture Notes in Computer Science, Vol. 2578. Springer-Verlag, Noordwijkerhout, The Netherlands (2002) 324-339

5. Johnson, M.K., Lyu, S., Farid, H.: Steganalysis in Recorded Speech. SPIE Symposium on Electronic Imaging San Jose CA (2005)

6. Hide4PGP Homepage: http://www.heinz-repp.onlinehome.de/Hide4PGP.htm 